\title{
Application of KM Platforms for Internal Operations
}

\author{
George Asimakopoulos, Vlasis Metaxas, Kostas Papadopoulos, and Elpida Metaxa
}

01 PLIROFORIKI S.A, 438 Acharnon St, 11143 Athens Greece

\begin{abstract}
This paper deals with these issues and reveals specific matters that are substantial for the transformation towards true e-government from today's digital representation of legacy processes. These issues have already been coped with in organizations that have transformed from a service based to a customer based model, due to regulatory changes. First is the use of KM in order to manage individual service users rather than operations. The second area is issue management, a technique that governmental organizations utilize in order to cope with public security matters.
\end{abstract}

\section{Introduction}

This paper deals with issues and reveals specific matters that are substantial for the transformation towards true e-government from today's digital representation of legacy processes. These issues have already been coped with in organizations that have transformed from a service based to a customer based model, due to regulatory changes.

First of these issues is the use of $\mathrm{KM}$ in order to manage individual service users rather than operations. The second area is issue management, a technique that governmental organizations utilize in order to cope with security matters.

The importance of KM in the public sector is easy to understand, if we consider that governmental processes are mostly about exchanging information with citizens. The major issue is that this information is not connected. This implies that although the governmental structure is handling information about citizens, it cannot transform it to knowledge, because this information does not reach as a whole the appropriate civil servant, but only as small bits of information. In other words, governmental organizations can not view citizens as an integral being but just as a part of many different processes. Under this perspective, KM could shift the government from viewing administrative processes to viewing individual citizens, through aggregating all information sources. This means that issues as information overload, interoperability, interfaces etc must be answered by any technology that will be selected.

Of course speaking about customers in the public sector is excessive. On the other hand though, as shown in the introduction, the public sector must shift towards a citizen based model from today's process or services based one. So there is much expertise to gain from organizations that have already shifted from their service based model to an individual end user model. After all, both citizens and customers are users of benefits that are provided from some kind of organizational structure. From

The original version of this chapter was revised: The copyright line was incorrect. This has been corrected. The Erratum to this chapter is available at DOI: 10.1007/978-3-540-44836-5_33

M.A. Wimmer (Ed.): KMGov 2003, LNAI 2645, pp. 268-273, 2003.

(C) Springer-Verlag Berlin Heidelberg 2003 
this point of view, any KM solutions that have been proven successful in such environments may easily be reused in governmental KM transformation.

Another governmental field where KM is very important is security. Awareness gives private enterprises the ability to react efficiently to environmental and market changes and thus gain competitive advantage. This awareness is more difficult to gain as competitors accrete and information sources regarding competitors, markets and customers are almost infinite. In the governmental sector though awareness is needed in order to establish security, either regarding external threats either threats that rise from inland agents. The fact that sources of threats have changed and multiplied means that vast amounts of information must be processed. The fact that information sources regarding threats are virtually infinite implies that manual monitoring of security relevant developments is not adequate. Governments need mechanisms that automatically can process vast amounts of information and forward specific bits of this information to individuals that can react to specific situations.

\section{Required KM Platform Characteristics}

The platform on which the solution could be built on must process automatically any kind of unstructured and structured information. Given the uninterrupted growth of unstructured information which is doubling every three months (Gartner), efficient processes that manage and extract value from such information are solely dependant on the ability to automate the tasks that previously, have been performed with manual labor.

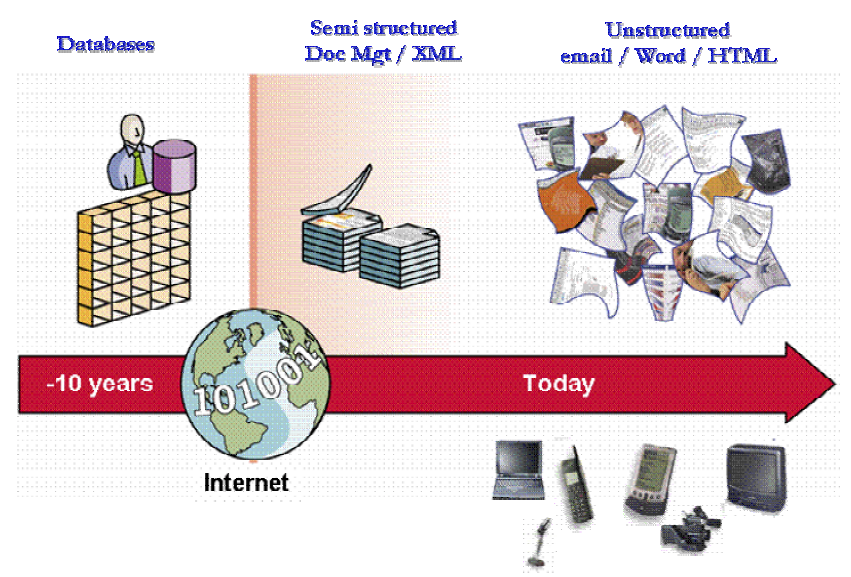

Fig. 1. Evolution of information texture requires automated processes

Following is an analytical description of the platform's desired functionality.

- Language independency: the software should be completely language independent. Usually most software relies on any intimate knowledge of English grammatical structure or that of any particular language. 
- Automated Content Aggregation and Management: the software should aggregate content from any data source. It should have the ability to read all widespread file formats and should be able to access all kinds of repositories. Furthermore, there should be no duplication of files into some new repository.

- Concept matching and automated hyperlinking: The software should accept a piece of content as an input and return references to conceptually related documents ranked by relevance or contextual distance.
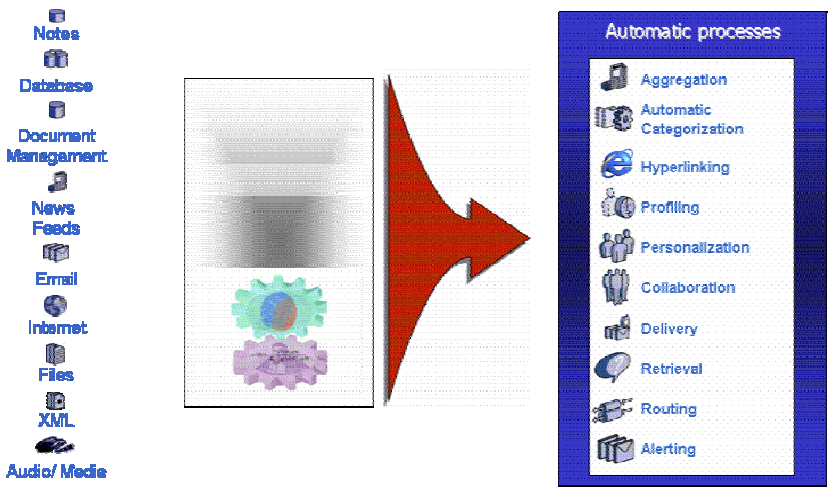

Fig. 2. Required automated processes

- Agent creation and retraining: The platform should accept a piece of content (a training phrase, document or set of documents) and return an encoded representation of the concepts, including each concept's specific underlying patterns of terms and associated probabilistic ratings.

- Agent matching and alerting: The software should accept an agent and return similar agents ranked by conceptual similarity. This is used to discover users with similar interests, or find experts in a field.

- Categorization: The software should accept a piece of content and return automatically categories ranked by conceptual similarity.

- Summarization: The software should accept a piece of content and return automatically a summary of the information containing the most salient concepts of the content.

- Retrieval: The software should support a Boolean term or natural language query and return a list of documents containing the terms ordered by contextual relevance to the query.

A software platform would be a very effective solution when processing of unstructured information is needed, as it reduces costs and processes any kind of data automatically.

\section{Case Study}

The case study that is presented here is from OTE. OTE is the operator that formerly was the only player in the Greek telecom market, as it was the organization with 
which the Greek government provided services to telecom users. Three years ago OTE transformed to the major but not only telecom player, as regulatory changes derived new alternative providers. This case study is useful because it reveals issues that must be coped with when a governmental organization shifts from a process based model to a customer or individual based model. The software used in this case was built by Autonomy.

Following is a description of how OTE coped with the two major issues presented in this paper: individual and issue management with the use of Autonomy's software.

\subsection{Issue Management}

First of all what was strongly needed by this Enterprise was a mechanism that would monitor the enterprise's business environment. This can be thought of as issue management, a technique that is widely used in the security field. Until the utilization of KM practices, this process was carried out by a group of people that constantly searched content that showed up in various sources such as internet sites, news feeds that the enterprise subscribed to and internal documents. As it can easily be understood, the results of the manual operation where not adequate, specially in a changing market where new and thus unknown competitors show up every day and change their commercial habits very frequently. First of all these workers could not cope with the vast amounts of information that overloaded them. This simply means that they could not read thoroughly every bit of information that showed up every moment. It is easy to understand that the automated content aggregation and management feature provided by Autonomy's software solved the problem of information overload. This is because this process was automated and thus executed thoroughly by computers.

The solution that was built on Autonomy's technology was the utilization of the concept matching feature in order to assign documents to specific categories. The approach was to describe the category and let the software match documents to each category description. In this way the enterprise now manages categories which are its main interest and not a vast set of documents. Another feature that expanded the enterprise's categorization capability was agent retraining. This feature allows the retraining of a category. This is done by using the concepts used in a document in order to describe a category.

Beyond category creation a major issue that must be solved is that of alerting. Every time a concept appears that is relevant to a users profile or an expertise description, the platform can alert the specific user. This implies that the enterprise can now respond to an issue, even if it has not been formally identified and assigned to individuals until the moment it appears. The alerting is accomplished with the software's agent alerting feature. This implies that as soon as an issue appears and not when someone finds out that it has occurred, categorizes the document and then inform a person that might not be able to react, because of the improper categorization that has been performed.

The areas in which this telecom provider utilized these issue management techniques were legal issues, competition awareness and benchmarked market leader observation. It is obvious though that these matters are encountered in every issue management initiative. 


\subsection{Individual Management}

The second KM application requested by the telecom operator was to build an ICT infrastructure that would enable the transition towards a customer based environment. The need to shift towards individual telecom users from the service centric view implied that regardless of services that are provided, the customer must be managed as a discrete individual that exhibits specific habits regarding the use of telecom and other adjunct services.

As said above, a major objective for e-government that has not been coped with until today is to accomplish the shift from the management of services to the management of civilians. The need for this shift though is not the same as it is for an enterprise that manages customers. But at the bottom line customers are individuals, so useful lessons can be taught from customer based transitions that are accomplished in the private sector. The public sector is built on a service based logic. This means that for each service provided, there is some kind of organizational structure in place. This structure exists only because the specific service is needed. This implies that every structure is ignorant about the other structures in place that provide different services. The utilization of ICT in the public sector until today has induced major wins in the governmental structure. The services described above have become faster, the localization distribution is not any more a drawback and paperwork is much less. The truth is that public services are improved and cheaper, because of the utilization of ITC. There is though one major drawback that keeps e-government away from the goal of treating citizens rather than services; this is that the use of ITC reproduced the organizational structure that was in place already. This means that all the ICT systems that are in place today do not exchange information. Information about citizens is stored in systems that are built to manage services.

The case of OTE again shows the strength that derives from the aggregation of multiple data sources when such transitions are needed. There where vast amounts of data regarding customers available on many different systems. These systems where managed by many different organizational units, ranging from the IT Division to the CEO's secretariat. This implies that it was very difficult to establish a organizational unit that could exploit this information. Of course, most of this information was unstructured. The result of this fact was that there was no way to see if a customer was profitable or not, because data was built in a way that allowed the monitoring of services and not individuals.

\section{Conclusions}

Appearance of citizen-centered technologies that make it possible for organizations to understand and respond to the needs of those they serve are influencing the information technology decisions made. The solution presented above supports:

- The growth of standards to allow different applications to exchange data, as it evolutes any kind of digital format, including voice and video. Virually any standard, existing or to be developed, is supported.

- The integration of documents and other unstructured information within applications. Local federal agencies are able to receive incoming information from 
the public that can be aggregated with the existing content, for example held on an environmental issue. Therefore enabling the authority to respond publicly in the appropriate manner. This can include external news feeds and newsgroups and can be particularly relevant with such public events as the US Presidential Elections.

- The arrival of network-based services that change the costs of using IT.

\section{Issues to be solved}

Although this approach seems appealing, there are various issues that must be resolved in order to maximize the benefits that arise from the presented solution. One of these issues is the utilization of unstructured information that resides on non electronic means of storage. Governmental organizations exist way before the use of ICT. This issue is partially solved, as the exploitation of ICT has lead to the digitalisation of most such data. The problem mostly occurs with data that follows an unstructured nature and is very rich in the term of concepts. The solution to this issue can not be different to the one that has been followed until today; data entry and/or scanning, OCR and manual error detection and correction.

Finally, there are some key points that show how the use of KM software that automates manual processes could push the transition to true e-government from where it is today. These points can be identified in the light of KM applications that have been deployed in large private sector organizations that utilize ICT infrastructure that is similar to that being used in the governmental structure. These main points are:

\section{Issue Management}

The development of government policy depends on the analysis of multiple information sources. Civil servants need accurate, timely information (in the form of Government reports, White Papers, etc.), which they then marshal in order to give rise to effective decision-making.

\section{Globalisation}

Many government operations will be spread throughout the world. In a military department's case, maintaining contact and ensuring that field personnel have access to information regardless of their position is vital. The importance of sharing information on a worldwide basis also highlights the importance of a scalable solution, Autonomy helps facilitate these operations.

\section{References}

1. Clarke T., Rollo C.: Corporate initiatives in knowledge management. Education \& Training, volume 43, number 4/5, 2001, p206-214. MCB University Press

2. Clarke S.: Knowledge Suite. Butler Viewpoint, February 1999

3. Harty J., Balla J., Andrews L.: White Paper on Functional Assessment of Autonomy products, Doculabs, December 1999. 